\title{
PITMAN ESTIMATORS: AN ASYMPTOTIC VARIANCE REVISITED*
}

\author{
A. NOVIKOV ${ }^{\dagger}$ AND N. KORDZAKHIA
}

(Translated by authors)

\begin{abstract}
We provide an analytic expression for the variance of ratio of integral functionals of fractional Brownian motion which arises as an asymptotic variance of Pitman estimators for a location parameter of independent identically distributed observations. The expression is obtained in terms of derivatives of a logarithmic moment of the integral functional of limit likelihood ratio process (LLRP). In the particular case when the LLRP is a geometric Brownian motion, we show that the established expression leads to the known representation of the asymptotic variance of Pitman estimator in terms of Riemann zeta-function.
\end{abstract}

Key words. Pitman estimators, fractional Brownian motion, integral functionals, Riemann zeta-function

DOI. $10.1137 / \mathrm{S} 0040585 \mathrm{X} 9798614 \mathrm{X}$

1. Introduction. The Pitman estimator [17] for the location parameter $\theta$ of independent random variables (r.v.'s) $\xi_{i}, i=1, \ldots, n$, with a density $f_{\theta}(x)=f(x-\theta)$ is given as

$$
\hat{\theta}_{n}=\frac{\int_{-\infty}^{\infty} u L_{u}^{(n)} d u}{\int_{-\infty}^{\infty} L_{u}^{(n)} d u}, \quad L_{u}^{(n)}:=\prod_{i=1}^{n} f\left(\xi_{i}-u\right) .
$$

The existence and admissibility of Pitman estimators have been discussed in [18]. The integrals in (1) exist for sufficiently large $n$ under the condition $\mathbf{E}\left|\xi_{i}\right|^{p}<\infty$ for some $p>0$. An exposition of some other important properties of Pitman estimators is presented in the textbook by Borovkov [4] (see also [20]).

The form of a limit distribution (as $n \rightarrow \infty)$ for the Pitman estimator has been derived by Ibragimov and Has'minskii [13], [15], who showed that under some regularity assumptions

$$
n^{\gamma}\left(\hat{\theta}_{n}-\theta\right) \stackrel{d}{\longrightarrow} \zeta
$$

in terms of convergence in distribution, where

$$
\zeta=\frac{\int_{-\infty}^{\infty} u L_{u} d u}{\int_{-\infty}^{\infty} L_{u} d u}
$$

$L_{u}$ is a limit likelihood ratio process, and the parameter $\gamma$ depends on the form of discontinuities of derivatives of the underlying density $f_{\theta}(x)$. In several important cases the structure of the process

$$
Y_{u}:=\log L_{u}
$$

has been described in [13], [15], and [16].

Let $W_{u}^{H}, u \in \mathbf{R}=(-\infty, \infty)$, be a fractional Brownian motion with the Hurst parameter $H \in(0,1]$. Recall that $W_{u}^{H}$ is a Gaussian continuous process with

$$
\mathbf{E} W_{u}^{H}=0, \quad \mathbf{E}\left|W_{u}^{H}-W_{s}^{H}\right|^{2}=|u-s|^{2 H}, \quad u, s \in \mathbf{R} .
$$

${ }^{*}$ Received by the editors September 1, 2011. This work was supported by the ARC Discovery grant DP120102398.

http://www.siam.org/journals/tvp/57-3/98614.html

†Steklov Mathematical Institute RAS, Gubkin St. 8, 119991 Moscow, Russia. Present address: Department of Mathematical Sciences, University of Technology, P.O. Box 123, Broadway, Sydney, NSW 2007, Australia (Alex.Novikov@uts.edu.au).

${ }_{\ddagger}$ Macquarie University, Sydney, NSW 2109, Australia (Nino.Kordzakhia@mq.edu.au). 
In [15] it has been shown that if $f_{\theta}(x)$ is a density of Weibull-like distributions, then

$$
Y_{u}=W_{u}^{H}-\frac{1}{2}|u|^{2 H}
$$

with $H \in\left(\frac{1}{2}, 1\right]$ and $\gamma=\frac{1}{2} H$ in (2).

Furthermore, the random variable $\zeta$ with $Y_{u}$ specified in (3), for the case $H=\frac{1}{2}$, occurs as a limit in a change-point problem for a Brownian motion (see [13], [15]) and (see [2]) for $H \in\left[\frac{1}{2}, 1\right]$ it occurs in estimation problems of ergodic diffusion processes in [16] and [12], and for $H \in\left(0, \frac{1}{2}\right)$ it also occurs in [9].

Note that, even in the case of $H=\frac{1}{2}$, the distribution of $\zeta$ and expressions for its even moments (higher than 2) are unknown in the explicit form.

In this paper we only discuss the problem of calculation of variance $\operatorname{Var}(\zeta)$ for the case (3) with $H \in\left[\frac{1}{2}, 1\right]$. The method developed in this paper employs auxiliary variables (see section 2) and it potentially can be used for obtaining analytical expressions and numerical approximations for other limiting processes, for example, when $L_{u}$ is a geometric Lévy process.

The special case $H=\frac{1}{2}$ in (3) was studied by several authors. In [14], using the simulation method it was obtained that

$$
\operatorname{Var} \zeta=19.5 \pm 0.5 \text {. }
$$

Golubev [11], using the Feyman-Kac formulae, found an analytical expression for $\operatorname{Var}(\zeta)$ in terms of integrals of products of Bessel functions and then, using numerical integration, obtained

$$
\operatorname{Var} \zeta=19.276 \pm 0.06 \text {. }
$$

Rubin and Song [19], using results of [11] and [21], found that

$$
\operatorname{Var} \zeta=16 \text { Zeta }[3]=19.2329 \ldots,
$$

where Zeta $[k]$ is the Riemann-zeta function.

In this paper we show (see Theorem 2 in section 2) that, for the case $H \in\left[\frac{1}{2}, 1\right]$ in (3), the value of $\operatorname{Var}(\zeta)$ can be expressed in terms of derivatives of the expectation of $\log \left(\int_{0}^{\infty}\left(e^{-m_{1} u} L_{u}+e^{-m_{2} u} L_{-u}\right) d u\right)$ with respect to parameters $m_{1}$ and $m_{2}$. Further, we will illustrate the application of this result for the case $H=\frac{1}{2}$, which leads to a relatively simple derivation of (4).

The following theorem implies the existence of moments of random variable $|\zeta|^{2 H}$, and it counts for its own sake.

THEOREM 1. If $H>\frac{\sqrt{5}-1}{4}$, then the random variable $|\zeta|^{2 H}$ is exponentially bounded; i.e., there exists a constant $\delta>0$ such that ${ }^{1}$

$$
\mathbf{E} \exp \left\{\delta|\zeta|^{2 H}\right\}<\infty .
$$

This result and some elements from its proof will be used in the proof of Theorem 2.

Proof. Set

$$
q_{t}=\frac{L_{t}}{\int_{-\infty}^{\infty} L_{u} d u}
$$

Since $q_{t}$ is a probability density function, by convexity argument, using Jensen's inequality, for any $\delta \geqq 0$, we have

$$
e^{\delta|\zeta|^{2 H}} \leqq c_{\delta}+\int_{-\infty}^{\infty} e^{\delta|t|^{2 H}} q_{t} d t
$$

with some constant $c_{\delta}$, (e.g., $c_{\delta}=0$ for the case $H \geqq \frac{1}{2}$ ).

\footnotetext{
${ }^{1}$ While the paper was in print we managed to improve the result of this theorem, showing that it is valid for all $H>0$.
} 
Since

$$
\left\{W_{u}^{H}, u \geqq 0\right\} \stackrel{d}{=}\left\{W_{-u}^{H}, u \geqq 0\right\},
$$

the random variable $\zeta$ has a symmetric distribution, and hence we have

$$
\mathbf{E} e^{\delta|\zeta|^{2 H}} \leqq c_{\delta}+2 \mathbf{E} \int_{0}^{\infty} e^{\delta t^{2 H}} q_{t} d t .
$$

Note

This implies

$$
\int_{0}^{1} e^{\delta t^{2 H}} q_{t} d t \leqq e^{\delta} \int_{0}^{\infty} q_{t} d t \leqq e^{\delta}
$$

$$
\mathbf{E} e^{\delta|\zeta|^{2 H}} \leqq c_{\delta}+2 e^{\delta}+2 \mathbf{E} \int_{1}^{\infty} e^{\delta t^{2 H}} q_{t} d t .
$$

To estimate $q_{t}$, note that by Jensen's inequality

$$
\int_{-\infty}^{\infty} L_{u} d u \geqq \int_{0}^{t} \exp \left\{Y_{u}\right\} d u \geqq t \exp \left\{\frac{1}{t} \int_{0}^{t} Y_{u} d u\right\}, \quad t>0,
$$

and hence

$$
q_{t} \leqq \frac{L_{t}}{\int_{0}^{t} L_{u} d u} \leqq \frac{1}{t} \exp \left\{Y_{t}-\frac{1}{t} \int_{0}^{t} Y_{u} d u\right\}, \quad t>0
$$

Thus we obtain

$$
\begin{aligned}
\mathbf{E} \int_{1}^{\infty} e^{\delta t^{2 H}} q_{t} d t & \leqq \mathbf{E} \int_{1}^{\infty} e^{\delta t^{2 H}} \exp \left\{-\frac{t^{2 H}}{2}+\frac{1}{t} \int_{0}^{t}\left(W_{t}^{H}-W_{u}^{H}+\frac{u^{2 H}}{2}\right) d u\right\} d t \\
& =\int_{1}^{\infty} \exp \left\{\delta t^{2 H}-\frac{H}{2 H+1} t^{2 H}\right\} \mathbf{E} \exp \left\{\frac{1}{t} \int_{0}^{t} W_{u}^{H} d u\right\} d t
\end{aligned}
$$

where the last equation holds due to the Fubini theorem and also due to the equalities

$$
\left\{W_{t}^{H}-W_{u}^{H}, u \in[0, t]\right\} \stackrel{d}{=}\left\{W_{t-u}^{H}, u \in[0, t]\right\}, \quad \int_{0}^{t} W_{t-u}^{H} d u \stackrel{d}{=} \int_{0}^{t} W_{u}^{H} d u .
$$

The random process $\int_{0}^{t} W_{s}^{H} d s$ is a zero-mean Gaussian process with the variance

$$
\begin{aligned}
\operatorname{Var} \int_{0}^{t} W_{s}^{H} d s=\mathbf{E}\left(\int_{0}^{t} W_{s}^{H} d s\right)^{2} & =\frac{1}{2} \int_{0}^{t} \int_{0}^{t}\left[s^{2 H}+u^{2 H}-|u-s|^{2 H}\right] d s d u \\
& =\frac{t^{2 H+2}}{2 H+2} .
\end{aligned}
$$

Therefore,

$$
\begin{aligned}
\mathbf{E} \int_{1}^{\infty} e^{\delta t^{2 H}} q_{t} d t & \leqq \int_{1}^{\infty} \exp \left\{\delta t^{2 H}-\frac{H}{2 H+1} t^{2 H}+\frac{1}{2 t^{2}} \operatorname{Var} \int_{0}^{t} W_{s}^{H} d s\right\} d t \\
& =\int_{1}^{\infty} \exp \left\{\delta t^{2 H}-t^{2 H}\left(\frac{H}{2 H+1}-\frac{1}{2} \frac{1}{2 H+2}\right)\right\} d t,
\end{aligned}
$$

where the last integral is finite for positive $\delta$ such that

$$
\delta<\frac{H}{2 H+1}-\frac{1}{2} \frac{1}{2 H+2}=\frac{4 H^{2}+2 H-1}{2(2 H+2)(2 H+1)} .
$$

It remains to note that $4 H^{2}+2 H-1>0$ when $H>\frac{\sqrt{5}-1}{4}=0.309 \ldots$.

Copyright (C) by SIAM. Unauthorized reproduction of this article is prohibited. 
2. The main result. Here we discuss the situation when the process $Y_{u}=\log L_{u}$ has the representation (3) with $H \in\left[\frac{1}{2}, 1\right]$.

For the proof of Theorem 2 we use the following representation:

$$
\operatorname{Var} \zeta=\frac{1}{2} \mathbf{E} \frac{\int_{-\infty}^{\infty} u^{2} L_{u} d u}{\int_{-\infty}^{\infty} L_{u} d u}
$$

This representation was proved by Golubev [11] for the case $H \in\left[\frac{1}{2}, 1\right]$.

It is convenient to use the following parametrized processes:

$$
\begin{array}{cc}
\beta_{1}(m)=\int_{0}^{\infty} e^{-m u} L_{u} d u, & \alpha_{1}(m)=\int_{0}^{\infty} u e^{-m u} L_{u} d u \\
\beta_{2}(m)=\int_{0}^{\infty} e^{-m u} L_{-u} d u, & \alpha_{2}(m)=\int_{0}^{\infty} u e^{-m u} L_{-u} d u
\end{array}
$$

where $m \geqq 0$ is an auxiliary parameter. Obviously, we obtain

$$
\begin{gathered}
\int_{-\infty}^{\infty} L_{u} d u=\beta_{1}(0)+\beta_{2}(0), \quad \int_{-\infty}^{\infty} u L_{u} d u=\alpha_{1}(0)-\alpha_{2}(0), \\
\alpha_{i}(m)=-\frac{\partial}{\partial m} \beta_{i}(m), \quad i=1,2,
\end{gathered}
$$

and

$$
\zeta=\frac{\alpha_{1}(0)-\alpha_{2}(0)}{\beta_{1}(0)+\beta_{2}(0)}
$$

In view of (5) we also have

$$
\alpha_{1}(0) \stackrel{d}{=} \alpha_{2}(0), \quad \beta_{1}(0) \stackrel{d}{=} \beta_{2}(0) .
$$

This implies that the random variable $\zeta$ has a symmetric distribution, and hence $\mathbf{E}(\zeta)=0$.

To formulate the main result we use the function

$$
g\left(m_{1}, m_{2}\right):=\mathbf{E} \log \left(\beta_{1}\left(m_{1}\right)+\beta_{2}\left(m_{2}\right)\right)=\mathbf{E} \log \int_{0}^{\infty}\left(e^{-m_{1} u} L_{u}+e^{-m_{2} u} L_{-u}\right) d u,
$$

where $m_{1}>0$ and $m_{2}>0$ are auxiliary parameters.

THEOREM 2. The function $g\left(m_{1}, m_{2}\right)$ is twice continuously differentiable and

$$
\operatorname{Var} \zeta=2 \lim _{m_{1} \rightarrow 0, m_{2} \rightarrow 0}\left[\frac{\partial^{2} g\left(m_{1}, m_{2}\right)}{\partial m_{1}^{2}}-\frac{\partial^{2} g\left(m_{1}, m_{2}\right)}{\partial m_{1} \partial m_{2}}\right] .
$$

Proof. First note that the function $g\left(m_{1}, m_{2}\right)$ is finite

$$
g\left(m_{1}, m_{2}\right) \leqq \mathbf{E} \int_{0}^{\infty}\left(e^{-m_{1} u} L_{u}+e^{-m_{2} u} L_{-u}\right) d u=\frac{1}{m_{1}}+\frac{1}{m_{2}}<\infty
$$

due to the inequality $\log x \leqq x, x>0$, and the equality $\mathbf{E} L_{u}=\mathbf{E}\left(L_{-u}\right)=1$. Obviously, $g\left(m_{1}, m_{2}\right)>-\infty($ see $(6))$.

The variance $\operatorname{Var}(\zeta)=\mathbf{E}\left(\zeta^{2}\right)<\infty$ (see Theorem 1), and in view of (8) we have

$$
\begin{aligned}
\operatorname{Var} \zeta=\mathbf{E}\left(\frac{\alpha_{1}(0)-\alpha_{2}(0)}{\beta_{1}(0)+\beta_{2}(0)}\right)^{2}= & -2 \mathbf{E} \frac{\alpha_{1}(0) \alpha_{2}(0)}{\left(\beta_{1}(0)+\beta_{2}(0)\right)^{2}}+\mathbf{E} \frac{\alpha_{1}(0)^{2}}{\left(\beta_{1}(0)+\beta_{2}(0)\right)^{2}} \\
& +\mathbf{E} \frac{\alpha_{2}(0)^{2}}{\left(\beta_{1}(0)+\beta_{2}(0)\right)^{2}}
\end{aligned}
$$

Note that the last two terms are equal due to the property of symmetry (9).

Copyright (C) by SIAM. Unauthorized reproduction of this article is prohibited. 
Further, for $m_{1}>0, m_{2}>0$, we have

$$
\begin{aligned}
\mathbf{E}\left(\frac{\alpha_{1}\left(m_{1}\right)-\alpha_{2}\left(m_{2}\right)}{\beta_{1}\left(m_{1}\right)+\beta_{2}\left(m_{2}\right)}\right)^{2}= & -2 \mathbf{E} \frac{\alpha_{1}\left(m_{1}\right) \alpha_{2}\left(m_{2}\right)}{\left(\beta_{1}\left(m_{1}\right)+\beta_{2}\left(m_{2}\right)\right)^{2}}+\mathbf{E} \frac{\alpha_{1}\left(m_{1}\right)^{2}}{\left(\beta_{1}\left(m_{1}\right)+\beta_{2}\left(m_{2}\right)\right)^{2}} \\
& +\mathbf{E} \frac{\alpha_{2}\left(m_{2}\right)^{2}}{\left(\beta_{1}\left(m_{1}\right)+\beta_{2}\left(m_{2}\right)\right)^{2}} .
\end{aligned}
$$

To find the first term in the right-hand side of (10), we note that the random functions $\beta_{i}(m)$ are continuously differentiable. By direct calculations we obtain

$$
Q\left(m_{1}, m_{2}\right):=\frac{\alpha_{1}\left(m_{1}\right) \alpha_{2}\left(m_{2}\right)}{\left(\beta_{1}\left(m_{1}\right)+\beta_{2}\left(m_{2}\right)\right)^{2}}=-\frac{\partial^{2} \log \left(\beta_{1}\left(m_{1}\right)+\beta_{2}\left(m_{2}\right)\right)}{\partial m_{1} \partial m_{2}}
$$

for $m_{1}>0, m_{2}>0$. Applying the inequality $x y \leqq x^{2}+y^{2}$, we have

$$
Q\left(m_{1}, m_{2}\right) \leqq\left(\frac{\alpha_{1}\left(m_{1}\right)}{\beta_{1}\left(m_{1}\right)}\right)^{2}+\left(\frac{\alpha_{2}\left(m_{2}\right)}{\beta_{2}\left(m_{2}\right)}\right)^{2}
$$

Now we show that the right-hand side of this inequality is uniformly bounded by an integrable random variable.

Set

$$
p_{t}(m)=\frac{e^{-m t} L_{t}}{\int_{0}^{\infty} e^{-m u} L_{u} d u} .
$$

Since $p_{t}(m)$ is a probability density function, by the Cauchy-Bunyakovsky inequality we have

$$
\left(\frac{\alpha_{1}(m)}{\beta_{1}(m)}\right)^{2}=\left(\int_{0}^{\infty} t p_{t}(m) d t\right)^{2} \leqq \int_{0}^{\infty} t^{2} p_{t}(m) d t
$$

To estimate $p_{t}(m)$ we apply the following inequalities:

$$
p_{t}(m) \leqq \frac{e^{-m t} L_{t}}{\int_{0}^{t} e^{-m u} L_{u} d u} \leqq \frac{L_{t}}{\int_{0}^{t} L_{u} d u} \leqq \frac{L_{t}}{t \exp \left\{(1 / t) \int_{0}^{t}\left(W_{u}^{H}-u^{2 H} / 2\right) d u\right\}}
$$

and thus obtain

$$
\begin{aligned}
\int_{0}^{\infty} t^{2} p_{t}(m) d t & \leqq \int_{0}^{\infty} t \exp \left\{-\frac{t^{2 H}}{2}+\frac{1}{t} \int_{0}^{t}\left(W_{t}^{H}-W_{u}^{H}+\frac{u^{2 H}}{2}\right) d u\right\} d t \\
& =\int_{0}^{\infty} t \exp \left\{-\frac{H}{2 H+1} t^{2 H}+\frac{1}{t} \int_{0}^{t}\left(W_{t}^{H}-W_{u}^{H}\right) d u\right\} d t=: Z .
\end{aligned}
$$

A similar estimate is valid for the second term $\left(\alpha_{2}(m) / \beta_{2}(m)\right)^{2}$ in (13).

Referring to the proof of Theorem 1, we obtain

$$
\mathbf{E} Z=\int_{0}^{\infty} t \exp \left\{-t^{2 H}\left(\frac{H}{2 H+1}-\frac{1}{2} \frac{1}{2 H+2}\right)\right\} d t<\infty
$$

for the case $H \geqq \frac{1}{2}$. This implies that the random function $Q\left(m_{1}, m_{2}\right)$ is uniformly bounded by the parameter-free integrable random variable $Z$.

Applying the expectation to both parts of (12) and well-known theorems about differentiability of expectations over parameters, we can conclude that the function $g\left(m_{1}, m_{2}\right)$ has a continuous mixed derivative and

$$
\mathbf{E} Q\left(m_{1}, m_{2}\right)=\mathbf{E} \frac{\alpha_{1}\left(m_{1}\right) \alpha_{2}\left(m_{2}\right)}{\left(\beta_{1}\left(m_{1}\right)+\beta_{2}\left(m_{2}\right)\right)^{2}}=-\frac{\partial^{2} g\left(m_{1}, m_{2}\right)}{\partial m_{1} \partial m_{2}} .
$$

To find the second and third terms in (11) (and, correspondingly, in (10)) a similar consideration is used. One can check by direct calculations that

$$
\frac{\partial^{2} \log \left(\beta_{1}\left(m_{1}\right)+\beta_{2}\left(m_{2}\right)\right)}{\partial m_{1}^{2}}=-\frac{\alpha_{1}\left(m_{1}\right)^{2}}{\left(\beta_{1}\left(m_{1}\right)+\beta_{2}\left(m_{2}\right)\right)^{2}}+\frac{\int_{0}^{\infty} u^{2} e^{-m_{1} u} L_{u} d u}{\beta_{1}\left(m_{1}\right)+\beta_{2}\left(m_{2}\right)}
$$

Copyright (C) by SIAM. Unauthorized reproduction of this article is prohibited. 
This implies

$$
\begin{aligned}
\frac{\partial^{2} g\left(m_{1}, m_{2}\right)}{\partial m_{1}^{2}} & =\mathbf{E} \frac{\partial^{2} \log \left(\beta_{1}\left(m_{1}\right)+\beta_{2}\left(m_{2}\right)\right)}{\partial m_{1}^{2}} \\
& =-\mathbf{E} \frac{\alpha_{1}\left(m_{1}\right)^{2}}{\left(\beta_{1}\left(m_{1}\right)+\beta_{2}\left(m_{2}\right)\right)^{2}}+\mathbf{E} \frac{\int_{0}^{\infty} u^{2} e^{-m_{1} u} L_{u} d u}{\beta_{1}\left(m_{1}\right)+\beta_{2}\left(m_{2}\right)}
\end{aligned}
$$

Similarly one can get

$$
\begin{aligned}
\frac{\partial^{2} g\left(m_{1}, m_{2}\right)}{\partial m_{2}^{2}} & =\mathbf{E} \frac{\partial^{2} \log \left(\beta_{1}\left(m_{1}\right)+\beta_{2}\left(m_{2}\right)\right)}{\partial m_{2}^{2}} \\
& =-\mathbf{E} \frac{\alpha_{2}\left(m_{2}\right)^{2}}{\left(\beta_{1}\left(m_{1}\right)+\beta_{2}\left(m_{2}\right)\right)^{2}}+\mathbf{E} \frac{\int_{0}^{\infty} u^{2} e^{-m_{2} u} L_{u} d u}{\beta_{1}\left(m_{1}\right)+\beta_{2}\left(m_{2}\right)}
\end{aligned}
$$

Combining all of the above equations we obtain

$$
\begin{aligned}
\mathbf{E}\left(\frac{\alpha_{1}\left(m_{1}\right)-\alpha_{2}\left(m_{2}\right)}{\beta_{1}\left(m_{1}\right)+\beta_{2}\left(m_{2}\right)}\right)^{2}= & -\frac{\partial^{2} g\left(m_{1}, m_{2}\right)}{\partial m_{1}^{2}}-\frac{\partial^{2} g\left(m_{1}, m_{2}\right)}{\partial m_{2}^{2}}+2 \frac{\partial^{2} g\left(m_{1}, m_{2}\right)}{\partial m_{1} \partial m_{2}} \\
& +\mathbf{E}\left(\frac{\int_{0}^{\infty} u^{2} e^{-m_{1} u} L_{u} d u}{\beta_{1}\left(m_{1}\right)+\beta_{2}\left(m_{2}\right)}+\mathbf{E} \frac{\int_{0}^{\infty} u^{2} e^{-m_{1} u} L_{u} d u}{\beta_{1}\left(m_{1}\right)+\beta_{2}\left(m_{2}\right)}\right)
\end{aligned}
$$

Applying inequality $(x-y)^{2} \leqq 2 x^{2}+2 y^{2}$, we have

$$
\left(\frac{\alpha_{1}\left(m_{1}\right)-\alpha_{2}\left(m_{2}\right)}{\beta_{1}\left(m_{1}\right)+\beta_{2}\left(m_{2}\right)}\right)^{2} \leqq 2\left(\frac{\alpha_{1}\left(m_{1}\right)}{\beta_{1}\left(m_{1}\right)}\right)^{2}+2\left(\frac{\alpha_{2}\left(m_{2}\right)}{\beta_{2}\left(m_{2}\right)}\right)^{2},
$$

where the right-hand side is uniformly bounded by the parameter-free integrable random variable $Z$ as shown above. Now by Lebesgue's dominated convergence theorem we obtain

$$
\begin{aligned}
\operatorname{Var} \zeta= & \lim _{m_{1} \rightarrow 0, m_{2} \rightarrow 0} \mathbf{E}\left(\frac{\alpha_{1}\left(m_{1}\right)-\alpha_{2}\left(m_{2}\right)}{\beta_{1}\left(m_{1}\right)+\beta_{2}\left(m_{2}\right)}\right)^{2} \\
= & -\lim _{m_{1} \rightarrow 0, m_{2} \rightarrow 0}\left(\frac{\partial^{2} g\left(m_{1}, m_{2}\right)}{\partial m_{1}^{2}}+\frac{\partial^{2} g\left(m_{1}, m_{2}\right)}{\partial m_{2}^{2}}-2 \frac{\partial^{2} g\left(m_{1}, m_{2}\right)}{\partial m_{1} \partial m_{2}}\right) \\
& +2 \mathbf{E} \frac{\int_{0}^{\infty} u^{2} L_{u} d u}{\beta_{1}(0)+\beta_{2}(0)} .
\end{aligned}
$$

On the other hand, due to (5), formula (7) can be rewritten as follows:

$$
\operatorname{Var} \zeta=\mathbf{E} \frac{\int_{0}^{\infty} u^{2} L_{u} d u}{\beta_{1}(0)+\beta_{2}(0)}
$$

To complete the proof we need to substitute the last expression into (15), taking into account the equality

$$
\lim _{m_{1} \rightarrow 0, m_{2} \rightarrow 0} \frac{\partial^{2} g\left(m_{1}, m_{2}\right)}{\partial m_{1}^{2}}=\lim _{m_{1} \rightarrow 0, m_{2} \rightarrow 0} \frac{\partial^{2} g\left(m_{1}, m_{2}\right)}{\partial m_{2}^{2}},
$$

and then we solve the resulting linear equation with respect to $\operatorname{Var}(\zeta)$. This completes the proof of Theorem 2 .

Remark. 1. Other forms of the limit process $L_{u}$ or, equivalently, the process $Y_{u}$, appear in the change-point problems for Poisson processes (see, e.g., [7]) and autoregressive processes [6]. In these settings the authors obtained the representation

$$
Y_{u}=J_{u}^{+} I\{u \geqq 0\}+J_{u}^{-} I\{u<0\},
$$

where $J_{u}^{+}$and $J_{u}^{-}$are independent Lévy processes with some specific Lévy measures for jumps.

Copyright (C) by SIAM. Unauthorized reproduction of this article is prohibited. 
The representation (7) holds for the case (16) as well, under the condition

$$
\mathbf{E} e^{z J_{u}^{-}}=\mathbf{E} e^{(1-z) J_{u}^{+}} \text {for all } z \in(0,1)
$$

(private communication of A. Gushchin, 2011).

2. One can see that the right-hand side of (7) can be written in the form

$$
\operatorname{Var} \zeta=-\frac{1}{2} \lim _{m \rightarrow 0} \frac{\partial \mathbf{E} \log \int_{-\infty}^{\infty} e^{-m u^{2}} L_{u} d u}{\partial m} .
$$

Thus, $\operatorname{Var}(\zeta)$ can be found when the expectation $\mathbf{E}\left(\log \left(\int_{-\infty}^{\infty} e^{-m u^{2}} L_{u} d u\right)\right)$ is known in the explicit form as a function of $m$. To our knowledge there are no results of this kind in the current literature (besides the trivial case $H=1$ ). However, there are many results on distributions of integral exponentials of the form $\int_{0}^{\infty} e^{-m u+J_{u}} d u$, where $J_{u}$ is a Lévy process; see, e.g., [3], [5], [10], and references therein.

3. Under some additional assumptions, there exist other representations for $\operatorname{Var}(\zeta)$. For example, if for some $\varepsilon>0$

$$
\mathbf{E} \log \int_{0}^{\infty} e^{\varepsilon u} L_{u} d u<\infty
$$

then

$$
\operatorname{Var} \zeta=\lim _{m \rightarrow 0} \frac{\partial^{2} \mathbf{E} \log \int_{-\infty}^{\infty} e^{m u} L_{u} d u}{\partial m^{2}} .
$$

The proof of this formula can be obtained similarly to the proof of Theorem 2 .

3. Calculation of $\operatorname{Var}(\zeta)$ for the case of $\boldsymbol{H}=\frac{1}{2}$. We present here an elementary derivation of the explicit formula (4) based on the result of Theorem 2 and the well-known result on the distribution of the exponential functional of a standard Brownian motion. For the case under consideration

$$
\beta_{1}(m)=\int_{0}^{\infty} e^{W_{u}^{1 / 2}-(1 / 2+m) u} d u
$$

and

$$
\mathbf{P}\left(\frac{1}{\beta_{1}(m)}<x\right)=\int_{0}^{x} \frac{2(2 y)^{2 m}}{\Gamma(2 m+1)} e^{-2 y} d y,
$$

i.e., $\beta_{1}(m)$ has the reciprocal Gamma distribution (see [8]):

$$
\frac{1}{\beta_{1}(m)} \stackrel{d}{=} \text { Gamma }[2 m+1,2]
$$

Note that due to the properties of a standard Brownian motion, the random variables $\beta_{1}\left(m_{1}\right)$ and $\beta_{2}\left(m_{2}\right)$ are independent. To find the function $g\left(m_{1}, m_{2}\right)$, first note

$$
\mathbf{E} \log \frac{1}{\beta_{1}(m)}=\int_{0}^{\infty} \log x \frac{2(2 x)^{2 m} e^{-2 x}}{\Gamma(2 m+1)} d x=\Psi(0,2 m+1)-\log 2
$$

for any $m \geqq 0$ (this can be verified using Mathematica), where the function $\Psi(n, z)$ is the $(n+1)$ th derivative of logarithm of the gamma-function $\Gamma(z)$ :

$$
\Psi(n, z)=\text { PolyGamma }[n, z]=\frac{d^{n+1}}{d z^{n+1}} \log \Gamma(z) .
$$

Due to (19) and the fact that the sum of independent Gamma random variables is a random variable with the distribution Gamma $[\cdot, 2]$, we have

$$
\frac{1}{\beta_{1}\left(m_{1}\right)}+\frac{1}{\beta_{2}\left(m_{2}\right)} \stackrel{d}{=} \text { Gamma }\left[2 m_{1}+2 m_{2}+2,2\right],
$$

Copyright (C) by SIAM. Unauthorized reproduction of this article is prohibited. 
and hence

$$
\mathbf{E} \log \left(\frac{1}{\beta_{1}\left(m_{1}\right)}+\frac{1}{\beta_{2}\left(m_{2}\right)}\right)=\Psi\left(0,2 m_{1}+2 m_{2}+2\right)-\log 2 .
$$

Besides,

$$
\begin{aligned}
\mathbf{E} \log \left(\beta_{1}\left(m_{1}\right)+\beta_{2}\left(m_{2}\right)\right) & =\mathbf{E} \log \left(\frac{1}{\beta_{1}\left(m_{1}\right)}+\frac{1}{\beta_{2}\left(m_{2}\right)}\right)-\mathbf{E} \log \frac{1}{\beta_{1}\left(m_{1}\right)}-\mathbf{E} \log \frac{1}{\beta_{2}\left(m_{2}\right)} \\
& =\Psi\left(0,2 m_{1}+2 m_{2}+2\right)-\Psi\left(0,2 m_{1}+1\right)-\Psi\left(0,2 m_{2}+1\right)+\log 2,
\end{aligned}
$$

and hence

$$
\begin{aligned}
\frac{\partial^{2}}{\partial^{2} m_{1}} \mathbf{E} \log \left(\beta_{1}\left(m_{1}\right)+\beta_{2}\left(m_{2}\right)\right) & =4 \Psi\left(2,2 m_{1}+2 m_{2}+2\right)-4 \Psi\left(2,2 m_{1}+1\right), \\
\frac{\partial^{2}}{\partial m_{1} \partial m_{2}} \mathbf{E} \log \left(\beta_{1}\left(m_{1}\right)+\beta_{2}\left(m_{2}\right)\right) & =4 \Psi\left(2,2 m_{1}+2 m_{2}+2\right) .
\end{aligned}
$$

Now we are ready to calculate $\operatorname{Var} \zeta$ using Theorem 2. We have

$$
\begin{aligned}
\operatorname{Var} \zeta & =\left.2\left[\frac{\partial^{2} g\left(m_{1}, m_{2}\right)}{\partial m_{1}^{2}}-\frac{\partial^{2} g\left(m_{1}, m_{2}\right)}{\partial m_{1} \partial m_{2}}\right]\right|_{m_{1}=0, m_{2}=0} \\
& =8 \Psi(2,2)-8 \Psi(2,1)-8 \Psi(2,2)=-8 \Psi(2,1) .
\end{aligned}
$$

To complete the derivation of the explicit formula (4) we need to note

$$
\Psi(k, 1)=(-1)^{k+1} k \text { ! Zeta }[k+1]
$$

for any $k=1,2, \ldots$ (see $[1$, formula 6.4.2]). Thus we have

$$
\operatorname{Var} \zeta=-8 \Psi(2,1)=16 \text { Zeta [3]. }
$$

Remark. One can check that condition (17) does hold, and it is also possible to obtain the last result using formula (18).

Acknowledgments. The authors thank Yuri Kutoyants for the suggestion to study the problem of evaluations of asymptotic variances of Pitman estimators. The authors are indebted to Julia Mishura and Alexander Gushchin for discussions and suggestions which improved the presentation of the paper.

\section{REFERENCES}

[1] M. Abramowitz and I. Stegun, Handbook of Mathematical Functions, 10th Printing, U.S. National Bureau of Standards, U.S. Government Printing Office, Washington, DC, 1972.

[2] J. Antoch and M. Huskova, Bayesian-type estimators of change points, J. Statist. Plann. Inference, 91 (2000), pp. 195-208.

[3] J. Bertoin And M. Yor, Exponential functionals of Lévy processes, Probab. Surv., 2 (2005), pp. 191-212.

[4] A. A. Borovkov, Mathematical Statistics, Gordon and Breach Science Publishers, Amsterdam, 1998.

[5] P. Carmona, F. Petit, And M. Yor, Exponential functionals of Lévy processes, in Lévy Processes, Birkhäuser, Boston, MA, 2001, pp. 41-55.

[6] H. Chan And Y. Kutoyants, On parameter estimation of threshold autoregressive models, Stat. Inference Stoch. Process., 15 (2012), pp. 81-104.

[7] S. Dachian And I. Negri, On compound Poisson processes arising in change-point type statistical models as limiting likelihood ratios, Stat. Inference Stoch. Process., 14 (2011), pp. 255271.

[8] D. Dufresne, The distribution of a perpetuity, with applications to risk theory and pension funding, Scand. Actuar. J., 1990, no. 1-2, pp. 39-79.

Copyright $@$ by SIAM. Unauthorized reproduction of this article is prohibited. 
[9] T. FuJII, An extension of cusp estimation problem in ergodic diffusion processes, Statist. Probab. Lett., 80 (2010), pp. 779-783.

[10] H. GJessing and J. Paulsen, Present value distributions with applications to ruin theory and stochastic equations, Stochastic Process. Appl., 71 (1997), pp. 123-144.

[11] G. K. Golubev, Computation of efficiency of maximum-likelihood estimate when observing a discontinuous signal in white noise, Problems Inform. Transmission, 15 (1979), pp. 61-69 (in Russian).

[12] A. A. Gushchin and U. Küchler, On estimation of delay location, Stat. Inference Stoch. Process., 14 (2011), pp. 273-305.

[13] I. Ibragimov and R. Has'Minskit, The asymptotic behavior of generalized Bayesian estimates, Dokl. Akad. Nauk SSSR, 194 (1970), pp. 257-260 (in Russian).

[14] I. Ibragimov and R. Has'MinskiI, Estimation of the parameter of a discontinuous signal in Gaussian white noise, Problems Inform. Transmission, 11 (1975), pp. 31-43 (in Russian).

[15] I. Ibragimov and R. Has'minskit, Statistical Estimation. Asymptotic Theory, Springer-Verlag, New York, Berlin, 1981.

[16] Y. Kutoyants, Statistical Inference for Ergodic Diffusion Processes, Springer-Verlag, London, 2004.

[17] E. J. G. Pitman, The estimation of the location and scale parameters of a continuous population of any given form, Biometrika, 30 (1939), pp. 391-421.

[18] S. C. Pont And C. J. Stone, Fisher information and the Pitman estimator of a location parameter, Ann. Statist., 2 (1974), pp. 225-247.

[19] H. Rubin And K. Song, Exact computation of the asymptotic efficiency of maximum likelihood estimators of a discontinuous signal in a Gaussian white noise, Ann. Statist., 23 (1995), pp. $732-739$.

[20] C. Stein, The admissibility of Pitman's estimator for a single location parameter, Ann. Math. Statist., 30 (1959), pp. 518-522.

[21] M. YoR, Exponential Functionals of Brownian Motion and Related Processes, Springer-Verlag, Berlin, 2001.

Copyright (c) by SIAM. Unauthorized reproduction of this article is prohibited. 